\title{
Chinese herbal medicine for acute upper respiratory tract infections and reproductive safety: A systematic review
}

\author{
Zengshu Huang ${ }^{1,2,3}$, Xinyao Pan ${ }^{1,2,3,}$, Jing Zhou ${ }^{1,2,3}$, Wing Ting Leung ${ }^{1,2,3}$, Chuyu Li $^{1,2,3}$, \\ Ling Wang ${ }^{1,2,3, *}$ \\ ${ }^{1}$ Laboratory for Reproductive Immunology, Hospital \& Institute of Obstetrics and Gynecology, Shanghai Medical College, Fudan \\ University, Shanghai, China; \\ ${ }^{2}$ The Academy of Integrative Medicine, Fudan University, Shanghai, China; \\ ${ }^{3}$ Shanghai Key Laboratory of Female Reproductive Endocrine-related Diseases, Shanghai, China.
}

\begin{abstract}
Summary Acute upper respiratory tract infections (AURTIs) are common and self-limited in people with normal immunity but sometimes lead to poor clinical outcomes under specific conditions such as pregnancy if not treated appropriately. Chinese herbal medicines (CHM), which are widely used to treat AURTIs, have proven to be effective in preclinical and clinical studies. This review focuses on the bioactivities of typical CHM and the adverse reactions they cause, and especially issues with reproductive safety when treating AURTIs. The main mechanisms for clinical efficacy may include anti-viral, anti-bacterial, anti-inflammatory, antipyretic, and immunomodulatory action as indicated by preclinical evidence. Most clinical trials indicate that CHM shortens the natural course of AURTIs and that it relieves related symptoms such as a fever, headaches, coughing, myalgia, a cold, sore throat, and a nasal obstruction. However, some CHM have a range of adverse effects and potentially affect reproduction from endocrinal secretion to embryo development while others do not. Therefore, clinical adverse reactions and preclinical studies on the toxicity of $\mathrm{CHM}$ are discussed. More reliable evidence is required to conclude that CHM are efficacious and safe for pregnant women with AURTIs. This review should help to promote advances in the research on and development of CHM as alternative treatments for AURTIs and offer insight into strategies to manage the safety of CHM during clinical use.
\end{abstract}

Keywords: Chinese herbal medicine, acute upper respiratory tract infections, efficacy, reproductive safety, mechanism

\section{Introduction}

Acute upper respiratory tract infections (AURTIs) refer to infections that involve tonsillitis, pharyngitis, laryngitis, sinusitis, otitis media, and the common cold. Viruses are the pathogens that most often cause AURTIs, while bacteria, fungi, and helminths are far less common. Human rhinoviruses are the

Released online in J-STAGE as advance publication March 31,2019 .

${ }^{\S}$ These authors contributed equally to this work.

* Address correspondence to:

Dr. Ling Wang, Obstetrics \& Gynecology Hospital of Fudan University, 419 Fangxie Road, Shanghai 200011, China.

E-mail: Dr.wangling@fudan.edu.cn most prominent causes (1), but other viruses such as adenovirus and influenza virus and bacteria have also been implicated. Symptoms manifest as sneezing, nasal congestion, a sore throat, coughing, a fever, and headaches, with varying degrees of severity. Most AURTIs are mild and self-limited. Occasionally, they can lead to poor clinical outcomes, and their complications may threaten the health of the infected. Severe consequences tend to occur in people with a specific level of immunity, such as pregnancy.

Pregnant women with AURTIs usually face more problems such as a higher risk of severe clinical outcomes and more limitations on medication they can take. Western medicines are commonly used to treat pregnant women with AURTIs, but there are questions about their safety due to adverse reactions. Ibuprofen 
is a popular fever and pain reliever but contraindicated in pregnant women (2). Potential fetal defects prevent doctors from prescribing dextromethorphan, an antitussive component added to common cold medications, to pregnant women. Perceiving Chinese herbal medicines (CHM) to be less toxic, pregnant women in China tend to choose CHM when given the choice between Western medicine and CHM. According to China: 2015 Expert consensus on the standardization of common cold medicines for special populations, CHM are largely marketed as over-thecounter medicines (2). Having analyzed a total of 33 Chinese patent medicines (CPMs) listed in the 2012 China National Essential Drug List for the treatment of the common cold, Chen et al. concluded that CPMs had a potential positive effect on the cure rate of the common cold (3). Numerous clinical trials have indicated that CHM are efficacious in treating AURTIs, but their mechanisms of action and the adverse reactions they cause remain unclear. Greater efficacy and unknown toxicity underscore the significance of proper use of CHM in light of special situations such as pregnancy.

This review describes well-known herbs and traditional Chinese herbal preparations, it explains their main role in treating AURTIs, and it summarizes advances in the study of their efficacy and safety, and especially in terms of reproductive safety.

\section{Mechanisms by which CHM treat AURTIs}

\subsection{Anti-viral action}

The viral replication cycle includes attachment, penetration, transcription, protein synthesis, assembly, and release (4). These steps provide strategies to screen for CHM that prevent or control infections of the respiratory tract with viruses (Table 1). As an example, researchers searching for influenza neuraminidase (NA) inhibitors found that Forsythiae Fructus, Lonicera japonica, and Scutellaria baicalensis displayed exceptional performance $(5,6)$. Radix Isatidis inhibits the hemagglutinin (HA) of the influenza virus in the early stages (7) and then retains the influenza viral ribonucleoprotein (vRNP) complex in the nucleus (8), thereby blocking viral replication.

\subsection{Anti-bacterial action}

CHM tends to act to prevent bacterial growth or to reduce the possibility of sepsis. The mechanisms are complicated and unclear but probably relate to microbial enzymes or other interactions that inactivate microbial adhesins. As an example, phenolic compounds of Lonicera japonica have bacteriostatic activity (9) that affects a broad spectrum of bacteria, including Bacillus cereus and Staphylococcus aureus
(10), by damaging the cytoplasmic membrane.

\subsection{Anti-inflammatory action}

Symptoms of AURTIs are associated with viral replication and the related cytokine cascade response. In addition to direct antiviral action, CHMs often target multiple components of the inflammatory response to decrease production of pro-inflammatory mediators and recruitment of leukocytes. Lonicera japonica attenuates the expression of TNF-a, IL-6, and iNOS via downregulation of nuclear factor- $\kappa \mathrm{B}(\mathrm{NF}-\kappa \mathrm{B})$ pathways, and it enhances the expression of IL-10 by increasing Sp1 phosphorylation (11). Forsythiae Fructus suppresses the activation of mitogen-activated protein kinase (MAPK) and NF- $\mathrm{BB}$ pathways to decrease the production of TNF-a, IL-1 $\beta$, IL-6, and myeloperoxidase (MPO) (12). Flos Lonicera, Forsythia Fructus, and Radix Platycodon combinations have synergistic antiinflammatory action by alleviating pathological changes in the respiratory system and by reducing inflammatory cytokines in bronchoalveolar lavage fluid (BALF) (13).

\subsection{Antipyretic action}

A fever, a common feature of infectious diseases, serves as a defense response to pathogens as well as injuries to vital organs. The mechanism of fever onset is acknowledged to be sequential production of complement and prostaglandin E2 $\left(\mathrm{PGE}_{2}\right)$, followed by the subsequent transfer of pyrogenic cytokines peripherally and centrally (14). The efficacy of volatile oils, crucial to the treatment of fever with Radix Bupleuri, has been verified in a rat model of fever. This action might be associated with declining cAMP in the hypothalamus and arginine vasopressin (AVP) in the ventral septal area (15). Interestingly, another well-known antipyretic herb, Folium Mori, has potent synergistic action by relieving fever and inflammation when combined with Flos Chrysanthemi in a proportion of only $1: 1(16)$.

\subsection{Immunomodulatory action}

Host innate and adaptive immune responses to pathogens play a role throughout the course of AURTIs. Overreaction of the host immune response may also lead to tissue damage and multi-organ injuries, which in turn may cause related diseases (4). CHM are able to optimize host-pathogen immune responses of patients with AURTIs. As an example, the supplement of Bu-Zhong-Yi-Qi-Tang (BZYQT) after intranasal vaccination against influenza increases the virus-specific IgA and IgG antibody titers in the nasal cavity and sera by enhancing upper respiratory mucosal and systemic immunity (17). Interferon-a $($ IFN- $\alpha$ ) increases in the BALF of mice. The stimulation 


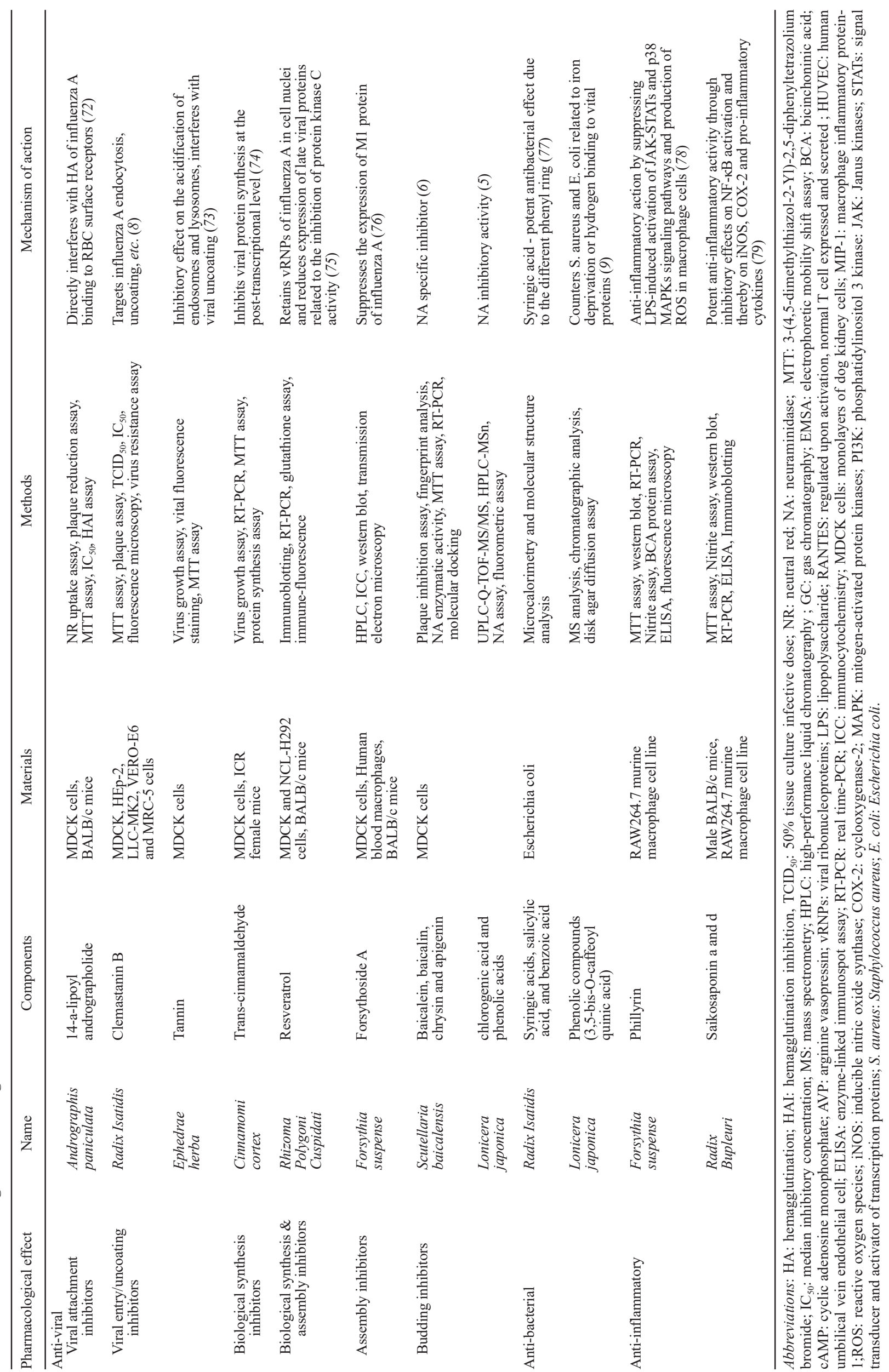




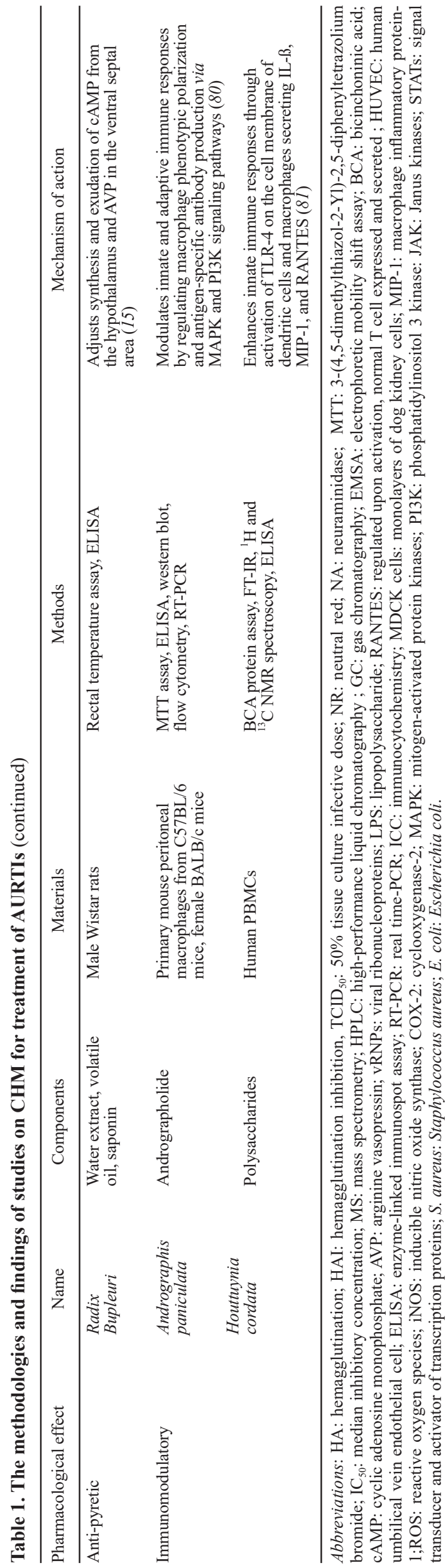

of both IFN- $\alpha$ production and antibody responses are possible explanations for the effect of BZYQT on "cold syndrome" (17).

\section{CHM in the treatment of AURTIs}

\subsection{Single herbs}

Frequently, the CHM that combat AURTIs are formulations rather than a single medicine, except for a few medicines such as Andrographis paniculate. According to a general search of a Chinese database, Flos Lonicerae Japonicae, Scutellaria baicalensis, Houttuynia cordata, Flos Chrysanthemi, Patrinia Herbae, Forsythia suspense, Radix Isatidis, Radix Bupleuri, Ephedra Herbae, Radix Glycyrrhizae, Ziziphus jujube, Rhizoma Polygoni Cuspidati, Folium Mori, Radix Platycodon, and Radix Puerariae are usually included in those formulations. A brief outline of the active component, pharmacological effect, and toxicity of the most commonly used single herbs is shown in Table 2

\subsection{CHM preparations}

Single Chinese herbs have proven ability to combat AURTIs. The synergistic effect of different individual herbs in traditional herbal formulations further enhances bioavailability and counteracts drug toxicity when combined with "monarch," "minister," "assistant," and "guide" components. Today, traditional herbal formulations are transformed into Chinese medicines through use of modern advanced pharmaceutical technology. This review provides evidence of the efficacy and safety of typical preparations in managing AURTIs (Table 3).

\subsubsection{Ge-gen-tang}

Ge-gen-tang (GGT) is a CHM formulation consisting of Puerariae Radix (Ge-gen), Ephedrae Herba (Mahuang), Cinnamomi Ramulus (Gui-zhi), Paeoniae Radix (Bai-shao), Glycyrrhizae Radix et Rhizoma preparata (Zhi-gan-cao), Zingiberis Rhizoma Recens (Sheng-jiang), and Jujubae Fructus (Da-zao) (18). GGT is of a great value in treating the common cold, fever, influenza, and even diarrhea in eastern Asia.

\subsubsection{Clinical findings}

A multicenter double-blind, parallel group, randomized controlled trial (RCT) of 240 patients with a cold in mainland China indicated that the GGT mixture is safe and efficacious (19). However, a study in Japan found that it did not significantly prevent the progression of cold symptoms (20). A point worth noting is that a study in Taiwan found that women who take GGT for relief 


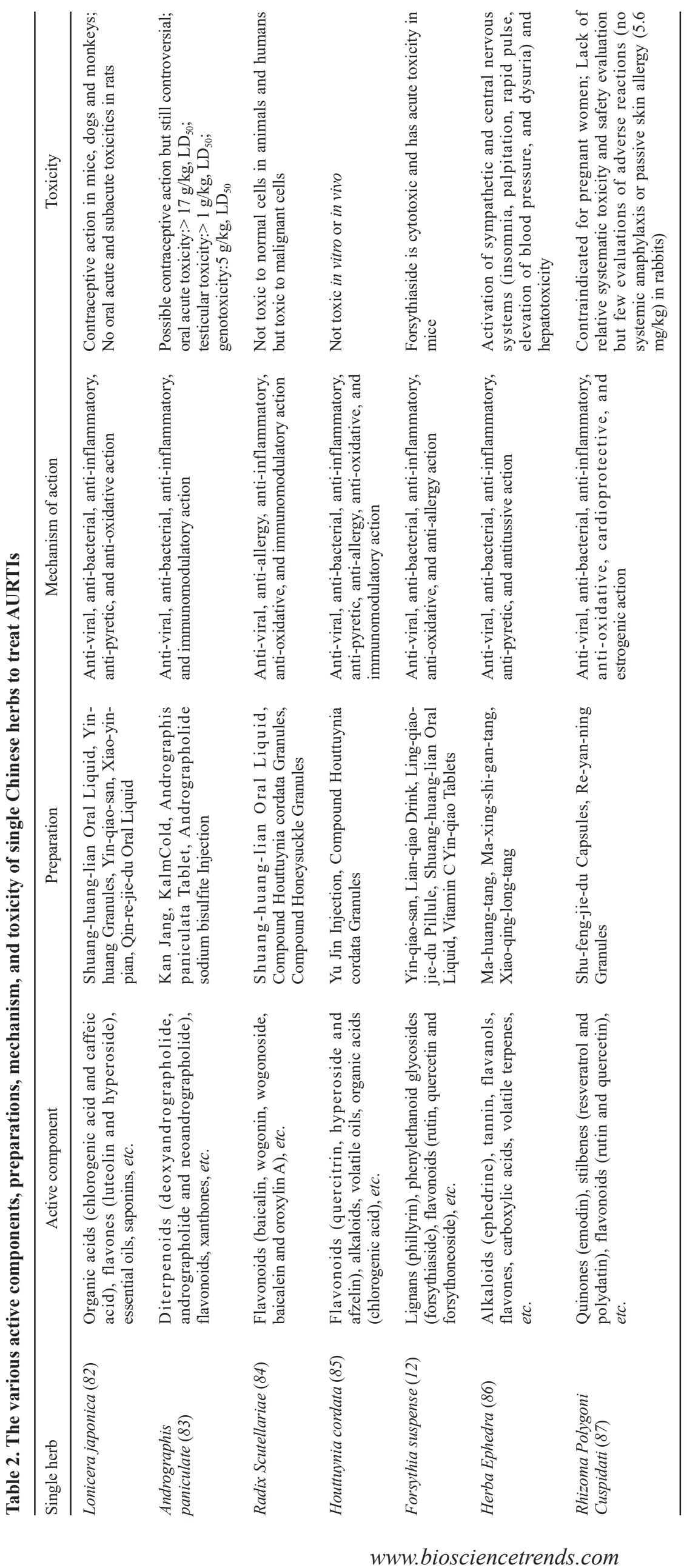




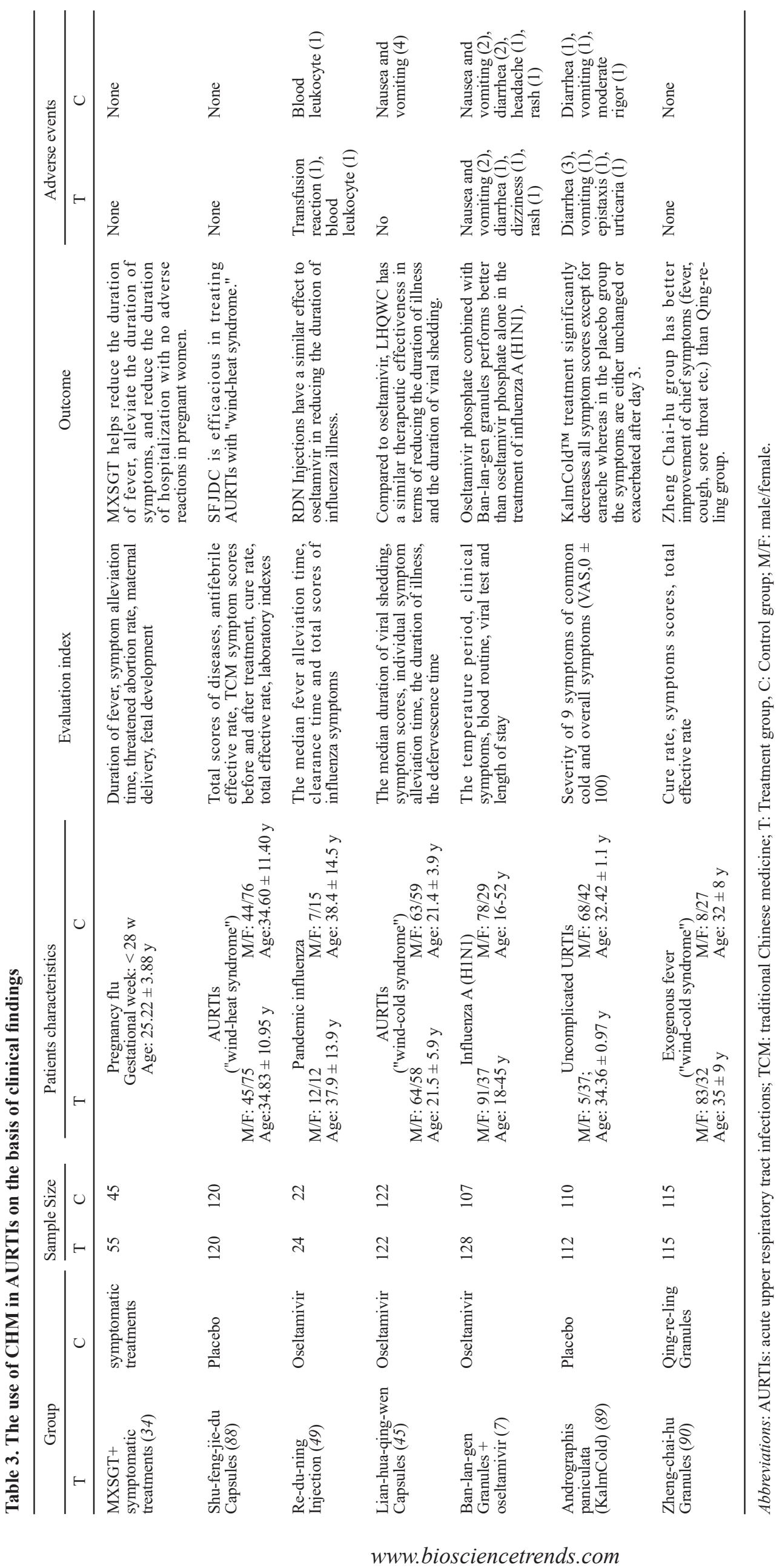


of respiratory discomfort are unexpectedly exposed to phytoestrogen generated by Ge gen (21). Nearly $5 \%$ of female users of Chinese medicines consumed cumulative doses of Ge gen above $60 \mathrm{~g}(21)$. Since little is known about the action of phytoestrogenic herbs in female patients, physicians need to be aware of threats to the female endocrine system when prescribing such herbs.

\subsubsection{Mechanism of action}

GGT stimulates the airway epithelium to secrete IFN- $\beta$ to counteract a viral infection before and after that infection, but it is more effective before infection (22). It disrupts influenza virus replication by directly blocking the virus-induced phosphatidylinositol 3-kinase (PI3K)/ Akt signaling pathway, which in turn causes retention of vRNPs in the nucleus (23). Promotion of the phagocytic activity of macrophages and an increase in the level of IL-12 in BALF contribute to a reduction in the virus yields as well (24).

\subsubsection{Shuang-huang-lian}

The Shuang-huang-lian (SHL) formulation, which consists of Lonicera japonica (Jin-yin-hua), Scutellaria baicalensis (Huang-qin), and Forsythia suspense (Lianqiao), generally has a satisfactory curative effect on AURTIs (25) and influenza (26) according to a large amount of laboratory and clinical data.

\subsubsection{Clinical findings}

A systematic review that evaluated the SHL injection (SHLI) in treating AURTIs concluded that it had potentially reduced the course of disease and relieved some cold symptoms like a fever and coughing (25). However, the poor methodology of the reviewed studies and poor reporting preclude reaching any definite conclusions on its clinical effectiveness. In another systematic review in 2016 that involved 21 studies (2,914 participants), meta-analysis revealed that SHLI was more efficacious at treating AURTIs than Western medicines (27).

\subsubsection{Mechanism of action}

SHL is reported as an effective agent against adenovirus and influenza virus (26). Human adenovirus III (HAdV3), a common pathogen causing AURTIs, is inhibited by SHL in a dose- and time-dependent manner, from viral attachment and internalization to replication, and is accompanied by a high level of IFN- $\alpha$ expression (28). In addition, SHL effectively attenuates lipopolysaccharide (LPS)-induced secretion of proinflammatory cytokines and oxidative stress by suppressing the p38- and ERK1/2-mediated AP-1 pathway in alveolar macrophages. Together, the reduction in neutrophil infiltration and excessive levels of inflammatory mediators alleviate pulmonary cellular injury (29). Screening of SHL in vitro revealed that chlorogenic acid is the main bioactive ingredient that specifically binds to $\beta 2$-adrenoceptor (30), which participates in smooth muscle relaxation and bronchodilation.

\subsubsection{Ma-xing-shi-gan-tang}

Ma-xing-shi-gan-tang (MXSGT) is a decoction consisting of Herba Ephedra (Ma-huang), Semen Armeniacae Amarum (Ku-xin-ren), Radix Glycyrrhizae (Gan-cao), and Gypsum Fibrosum (Shi-gao). Although MXSGT and Ma-huang-tang (MHT) differ in only one herb, both are conventional formulations for sweating, asthma, and febrile diseases such as influenza-like illness $(31,32)$. The corresponding "syndromes" (exogenous diseases) they treat according to traditional Chinese medicine (TCM) differ substantially.

\subsubsection{Clinical findings}

In an RCT involving 410 patients in 11 hospitals in 4 provinces of China, oseltamivir and MXSGT - Yinqiao-san (MXSGT-YQS), alone and in combination, reduced the duration of fever and effectively lowered severity scores for other symptoms in patients with an H1N1 influenza infection (33). Another RCT involving 100 patients was conducted to observe the effect of MXSGT in treating influenza during pregnancy (34). The control group $(n=55)$ received symptomatic treatment and/or intravenous penicillin $\left(8 \times 10^{6} \mathrm{U}\right)$ for 3 days if infected. The treatment group $(n=45)$ received MXSGT in addition to the same treatment as that of the control group. Flu therapy was more efficacious when combined with MXSGT, and the treatment group also had a lower threatened abortion rate than that of the control group. Follow-up of the treatment group revealed no adverse pregnancy outcomes while results for the control group were lost. Although there were significant differences between the two groups, this $\mathrm{RCT}$ did not constitute sufficient evidence.

\subsubsection{Mechanism of action}

MXSGT acts in several ways against human influenza A viruses in vitro by: 1) reducing viral uptake through damage of the viral ultrastructure; 2) inhibiting viral entry by inactivation of the PI3K/Akt signaling pathway; and 3) impairing the synthesis of both viral RNA and protein (35). Its extract relieves hyperthermic syndrome in rats via the synergic effects of Gypsum and Ephedra and through the modulation of $\mathrm{PGE}_{2}$ synthesis in the hypothalamus-adrenal gland axis (36). Early on, MXSGT has anti-inflammatory action by 
inhibiting neutrophil infiltration into the airway. It further attenuates lung microvascular hyperpermeability and decreases the number of leukocytes adhering to lung venules (37). Moreover, the antitussive action of MXSGT is dose-dependent property; it suppresses bronchial contractions induced by acetylcholine/ histamine (36).

\subsubsection{Shu-feng-jie-du Capsules}

Shu-feng-jie-du Capsules (SFJDC) have been extensively used to treat AURTIs and acute lung injury (ALI) for over 30 years in China. The capsules consist of 8 herbs: Rhizoma Polygoni Cuspidati (Hu-zhang), Fructus Forsythiae (Lian-qiao), Radix Isatidis (Banlan-gen), Radix Bupleuri (Chai-hu), Herba Patriniae (Bai-jiang-cao), Herba Verbenae (Ma-bian-cao), Rhizoma Phragmitis (Lu-gen), and Radix Glycyrrhizae (Gan-cao) (38).

\subsubsection{Clinical findings}

A multicenter, open-label phase IV clinical trial on SFJDC found that they were efficacious in treating AURTIs ("wind-heat syndrome"), with $0.03 \%$ of patients suffering an adverse drug reaction (ADR) (39). After 3 days of treatment, the cure rate was $40.23 \%$ and the total efficacy was $87.40 \%$. The median time for onset and duration of defervescence were 4.50 hours and 20.00 hours, respectively (39). According to an RCT, SFJDC alleviate acute suppurative tonsillitis, with a shorter duration of fever and pharyngeal purulence, greater shrinkage of the tonsils, and greater safety (40).

\subsubsection{Mechanism of action}

In vitro, SFJDC repress $\mathrm{H} 1 \mathrm{~N} 1$ (41), respiratory syncytial virus (RSV), coxsackie virus B3 (CoxB3), and herpes simplex virus 1 (HSV-1); SFJDC are less potent than ribavirin but also less cytotoxic (42). SFJDC are far more potent than Qing-kai-ling granules but weaker than ceftriaxone sodium injection in fighting 6 different bacteria (42). SFJDC protect against ALI by suppressing the MAPK/NF- $\kappa$ B pathway in vivo (43). Proteomic analysis of a rat model of LPS-induced ALI revealed that the anti-inflammatory and immunomodulatory actions of SFJDC are the results of action on the core regulator protein AKT1

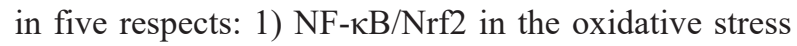
response; 2) the MAPK (ERK1/2 and JNK) signaling pathway; 3) p53/Bcl-2/Caspase in apoptosis; 4) AKT1/ SOCS1 in negative feedback to TLR4 signaling; 5) and AKT/mTOR signaling (38).

\subsubsection{Other preparations}

A number of new compound preparations that are derived from traditional formulations have come onto the market. Lian-hua-qing-wen Capsules (LHQWC) are an example that was developed from two conventional CHM formulae - MXSGT and YQS. LHQWC provide broad-spectrum protection against influenza viruses by restricting viral proliferation in the early stages, with an $\mathrm{IC}_{50}$ ranging from $0.35 \mathrm{mg} / \mathrm{mL}$ to $2 \mathrm{mg} / \mathrm{mL}$, and by efficiently blocking the nuclear export of vRNP (44). In addition, a virus-induced inflammatory reaction is alleviated by suppression of the NF-kB signaling pathway in a dose-dependent manner (44). In a doubleblind RCT involving 244 patients with influenza A, LHQWC performed similar to oseltamivir in reducing the duration of illness and viral shedding and in relieving the severity of illness and symptoms including fever, cough, sore throat, and fatigue (45).

Re-du-ning injection (RDNI) is another modern patent CHM prepared with an extract purified from Artemisia annua (Qing-hao), Gardenia jasminoides Ellis (Zhi-zi), and Flos Lonicerae (Jin-yin-hua). Previous studies indicated that RDNI inhibits human rhinovirus in vitro in three ways: inactivating the virus, interfering with viral proliferation, and blocking the interaction of infected cells (46). In addition to regulation of the host defense system (47), it has antipyretic action by reducing cAMP in the hypothalamus and MPO in the lungs of mice (48). Moreover, it shortens the clinical course of influenza, it alleviates fever, it reduces fever clearance time, and it alleviates all influenza symptoms (49). An RDNI infusion may be an effective alternative for treatment of influenza in adults, possibly with fewer adverse effects than oseltamivir (49). However, the clinical use of RDNI needs to be monitored to preclude off-label use, especially overdosing, improper dilution and overindication (50).

\section{Adverse reactions and reproductive safety}

\subsection{General adverse reactions to CHM}

The reasons why adverse reactions to CHM occur vary from inherent herbal toxicity, excessive doses of herbs, drug-herb interactions, anaphylaxis to coexisting diseases (51). Surprisingly, allergic reactions (skin itch, dermatitis and anaphylactic shock) occur in most cases of adverse reactions to CHM (52). ADR reports that are related to $\mathrm{CHM}$ injections account for $>50 \%$ of all ADR reports related to $\mathrm{CHM}$, and the percentage has been rising annually (53). Considerable attention is paid to the hepatic damage caused by CHM, but different organs may be affected (Tables 2 and 3 ). Some herbs may have no direct toxicity but cause harmful drug interactions, which are mainly associated with cytochrome P450(CYP)-linked drug metabolism. If CHM are used with other drugs, their curative effect might be hampered or their toxicity increased, or both. 
One herb can sometimes have multiple impacts in terms of adverse reactions. As an example, oral intake of andrographolide extracted from Andrographis paniculata likely leads to reduced metabolic activity of intestinal CYP3A4 (54) and induces nephrotoxicity associated with activation of oxidative and endoplasmic reticulum stress (55).

\subsection{Reproductive safety of $C H M$}

In addition to considering general adverse reactions, more attention needs to be paid to the reproductive safety of pregnant women. There is no doubt that pregnancy is a dynamic process for both the mother and fetus. This complicated situation requires the consideration of the continued health of the mother, embryo-fetal development, and prenatal growth during different gestational stages. The placenta, a key avenue for the maternal-fetal transfer of substances, has alterations in transport proteins (56) and expression of biotransformation enzymes (57). Consequently, drug permeability, metabolism, and clearance differ from the early to late period (58). As an example, chemicals like chlorinated insecticides, probably used to farm herbs, affect oxytocin, testosterone, oestradiol, and prostaglandin secretion of ovarian and uterine cells, interfering with fertilization as well as with myometrial contractions (59). Accordingly, the reproductive organs could be affected by contaminated CHM.

Temporal variations in pharmacokinetics (PK) and pharmacodynamics (PD) during gestation are not limited to the reproductive system. A previous study found that gestation influences the systematic PK profile of certain Chinese herbs such as Puerariae Radix (puerarin), with different levels during different stages in pregnant rats (60). Dynamic changes in CYPmediated drug metabolism in the body of pregnant women have also been reported (61). Much more should be known about the reproductive safety of CHM. This can be achieved by monitoring the magnitude of variations in physiologic and morphological parameters of the reproductive system and all relevant changes related to drug therapy during pregnancy that may affect maternal-fetal health.

\subsection{Status of research}

In fact, most clinical studies of CHM preparations for treatment of AURTIs exclude pregnant women, who are not often exposed to the uncertain safety risks of medicines in clinical trials in order to avoid further complications. As a consequence, there is a lack convincing clinical evidence of the reproductive safety of many CHM preparations. There are only a few studies of clinical outcomes of use of single herbs and preparations by pregnant women (34) and studies of reproductive toxicity $(62)$.
As an example, no study has examined the toxicity of a daily dose (6-15 g) of Forsythiae Fructus thus far (Pharmacopoeia Commission of the PRC, 2015). Administration of forsythoside gelsiccation powder to SD rats yielded negative results in terms of teratogenicity (63). However, one of the active constituents of Forsythiae Fructus, forsythiaside, caused acute toxicity in mice $\left(\mathrm{IC}_{50}=1.98 \mathrm{~g} / \mathrm{kg}\right)(12)$.

The metabolic process of a single herb and be determined and its target organ can be identified, but studying adverse reactions to preparations is much more difficult due to the multiple components and targets involved. Preparations intensify or diminish the toxicity of single herbs. According to a cytotoxicity assay, SHL is slightly less toxic than a mixture of its active ingredients - chlorogenic acid, baicalin, and forsythia glycosides A (28).

Adverse reactions to SHL have often been studied. Of the different dosage forms such as oral liquids, granules, and injections, SHLI caused most cases of adverse reactions (64). According to a systematic review (64), ADRs caused by SHLI are mainly skin allergic reactions and gastrointestinal reactions. Involved systems or organs are, in decreasing order: the skin, the digestive system, general reactions, the respiratory system, and other systems or organs. In addition, there is an increased risk of ADRs induced by combining SHLI and other drugs, and especially antibiotics. Nearly all cases of death were caused by anaphylactic shock (65) induced by $\mathrm{C} 5$ activation and the subsequent release of histamine, which is largely due to chlorogenic acid (66).

In 2018 , China's State Food and Drug Administration announced the prohibition of the use of SHLI by children under 4 years of age and pregnant women, but instructions on other dosage forms still indicate that use of SHLI by pregnant or nursing women should be under a physician's direction. Clearly, in-depth studies on the reproductive safety of SHL need to continue in light of previous studies. In addition to its indication for AURTIs, one herb in SHL, Radix Scutellariae, is listed as an herb for prevention of miscarriage. Baicalin, one of its active components, has anti-abortifacient action (67), and it reverses trophoblast apoptosis in the treatment of preeclampsia (68). However, several individual cases of hepatotoxicity after taking Radix Scutellariae have been reported (69). Moreover, a study of SHL as a frozen powder provided evidence that baicalein and baicalin exchange can occur in the maternal-fetal interface but that it does not affect the contractile activity of human uterine smooth muscle strips in vitro (70). A later study indicated weak toxicity to the maternal side of the placental barrier with no toxicity to the fetal side in vitro (71). Although the toxicological and protective roles of the placental barrier with respect to drug detoxification and transporter-controlled protection of the fetus have been 
intensively examined (57), little work has been done to elucidate the detailed mechanism at the molecular biological level.

\section{Conclusion}

There is no doubt that CHM play important roles in treating AURTIs by controlling and preventing those infections, limiting the economic burden in comparison to oseltamivir, and benefiting patients with poor tolerance to Western medicines in clinical practice. More advanced technology and strategies allow identification of active CHM and CHM-derived compounds from a vast body of traditional CHM via high-throughput screening, and they allow determination of the mechanisms by which CHM treat AURTIs (4). Nonetheless, some problems still limit the use of CHM to treat AURTIs, such as inconsistent clinical efficacy and the insufficient evaluation of safety.

Since there are still many herbs and preparations for treatment of AURTIs with either contradictory results or no systematic evaluation of efficacy and safety, and especially reproductive safety, attention must be paid to conducting more well-designed clinical trials and studies to help avoid the misuse of CHM. Although some single herbs or formulations are cited as suitable for pregnant women, more reliable evidence combining preclinical and clinical data should be obtained. Indepth research at the molecular level is needed to reveal underlying pharmacological mechanisms of efficacy and reproductive safety. Moreover, the range of teratogenicity testing should be extended to fertility, sexual maturity, and even organ changes in pregnancy.

Patients may have reduced tolerance and, therefore, more toxicity studies should be conducted on both normal and ill subjects. Given differences between animals and humans, more clinical studies on the safety of CHM during pregnancy are required before CHM can ultimately be used clinically. These efforts may help to formulate strategies to prevent ADRs by adding antagonist drugs or by optimizing the dose, thus minimize risk and maximizing benefit for the mother and fetus in clinical settings.

\section{Acknowledgements}

This work was supported by grants from the National Natural Science Foundation of China (no. 31571196 to Ling Wang and no. 30801502 to Ling Wang), the Program to Guide Medicine ("Yixueyindao") of the Shanghai Municipal Science and Technology Commission (no. 18401902200 to Ling Wang and no. 15401932200 to Ling Wang), the Shanghai Program for Support of Leading Disciplines-Integrative Medicine (no. 20180101 and no. 20150407), the Research Foundation ("CR Sanjiu") of Obstetrics \& Gynecology committee of Chinese Association of
Integrated Traditional Chinese and Western Medicine (CR1901FC01 to Ling Wang), the Shanghai Committee of the China Democratic League (no. 02054 to Ling Wang), the FY2008 JSPS Postdoctoral Fellowship for Foreign Researchers (P08471 to Ling Wang), and the Shanghai Pujiang Program (no. 11PJ1401900 to Ling Wang).

\section{References}

1. Passioti M, Maggina P, Megremis S, Papadopoulos NG. The common cold: Potential for future prevention or cure. Curr Allergy Asthma Rep. 2014; 14:413.

2. China: Expert consensus on the standardization of common cold medicines for special populations. International Journal of Respiration. 2015;35:1-5. (in Chinese)

3. Chen W, Liu B, Wang LQ, Ren J, Liu JP. Chinese patent medicines for the treatment of the common cold: A systematic review of randomized clinical trials. BMC Complementary Altern Med. 2014; 14:273-280.

4. Li T, Peng T. Traditional Chinese herbal medicine as a source of molecules with antiviral activity. Antiviral Res. 2013; 97:1-9.

5. Gamaleldin Elsadig Karar M, Matei MF, Jaiswal R, Illenberger S, Kuhnert N. Neuraminidase inhibition of dietary chlorogenic acids and derivatives - Potential antivirals from dietary sources. Food Funct. 2016; 7:20522059.

6. Hour MJ, Huang SH, Chang CY, Lin YK, Wang CY, Chang YS, Lin CW. Baicalein, ethyl acetate, and chloroform extracts of Scutellaria baicalensis inhibit the neuraminidase activity of pandemic 2009 H1N1 and seasonal influenza A viruses. Evid Based Complement Alternat Med. 2013; 2013:750-803.

7. Tu B, Nie W, Ding PP, Li FY, Chen WW, Zhou ZP, Zhang X, Fan R, Huo DD, Zhao M. Efficacy of treatment of influenza A (H1N1) with oseltamivir phosphate and isatis root granules. Medical Journal of the Chinese People's Armed Police Forces. 2013; 24:465-467+470. (in Chinese)

8. Yang Z, Wang Y, Zheng Z, Zhao S, Zhao J, Lin Q, Li C, Zhu Q, Zhong N. Antiviral activity of Isatis indigotica root-derived clemastanin $\mathrm{B}$ against human and avian influenza A and B viruses in vitro. Int J Mol Med. 2013; 31:867-873.

9. Xiong J, Li S, Wang W, Hong Y, Tang K, Luo Q. Screening and identification of the antibacterial bioactive compounds from Lonicera japonica Thunb. leaves. Food Chem. 2013; 138:327-333.

10. Shan B, Cai YZ, Brooks JD, Corke H. The in vitro antibacterial activity of dietary spice and medicinal herb extracts. Int J Food Microbiol. 2007; 117:112-119.

11. Kao ST, Liu CJ, Yeh CC. Protective and immunomodulatory effect of flos Lonicerae japonicae by augmenting IL-10 expression in a murine model of acute lung inflammation. J Ethnopharmacol. 2015; 168:108115 .

12. Wang Z, Xia Q, Liu X, Liu W, Huang W, Mei X, Luo J, Shan M, Lin R, Zou D, Ma Z. Phytochemistry, pharmacology, quality control and future research of Forsythia suspensa (Thunb.) Vahl: A review. J Ethnopharmacol. 2018; 210:318-339. 
13. Li YH, Zheng FJ, Huang Y, Zhong XG, Guo MZ. Synergistic anti-inflammatory effect of Radix Platycodon in combination with herbs for cleaning-heat and detoxification and its mechanism. Chin J Integr Med. 2013; 19:29-35.

14. Roth J, Blatteis CM. Mechanisms of fever production and lysis: Lessons from experimental LPS fever. Compr Physiol. 2014; 4:1563-1604.

15. Jin GT, Li B, Wang SR. Experimental study on material basis, efficacy and mechanism of antipyretic effect of Bupleuri Radix. Journal of Chengdu University of Traditional Chinese Medicine. 2013; 36:28-30. (in Chinese)

16. Qin XJ, Ma C. Experimental study on pyretolysis mechanism of compatible application of Folium Mori and Flos Chrysanthemi. Journal of New Chinese Medicine. 2013; 45:133-135.

17. Kiyohara H, Nagai T, Munakata K, Nonaka K, Hanawa T, Kim SJ, Yamada H. Stimulating effect of Japanese herbal (kampo) medicine, hochuekkito on upper respiratory mucosal immune system. Evid Based Complement Alternat Med. 2006; 3:459-467.

18. Yan Y, Chai CZ, Wang DW, Yue XY, Zhu DN, Yu BY. HPLC-DAD-Q-TOF-MS/MS analysis and HPLC quantitation of chemical constituents in traditional Chinese medicinal formula Ge-Gen Decoction. J Pharm Biomed Anal. 2013; 80:192-202.

19. Song HN, Mao ZF, Han DF, Xiang N, Li RL. Randomized double-blind controlled trial on Gegentang mixture in treatment of cold. Clinical Focus. 2005; 20:313-315.

20. Kitamura H, Urano H, Ara T. Preventive effects of a Kampo medicine, Kakkonto, on inflammatory responses via the suppression of extracellular signal-regulated kinase phosphorylation in lipopolysaccharide-treated human gingival fibroblasts. ISRN Pharmacol. 2014; 2014:1-7.

21. Wu CT, Tzeng JN, Lai JN, Tsan SH, Wang JD. Prescription profile of Chinese herbal products containing coumestrol, genestein, and/or daidzein among female users: An analysis of national health insurance data in Taiwan between 1997 and 2007. Chin Med. 2012; 7:22.

22. Chang JS, Wang KC, Shieh DE, Hsu FF, Chiang LC. GeGen-Tang has anti-viral activity against human respiratory syncytial virus in human respiratory tract cell lines. J Ethnopharmacol. 2012; 139:305-310.

23. Wu MS, Yen HR, Chang CW, Peng TY, Hsieh CF, Chen CJ, Lin TY, Horng JT. Mechanism of action of the suppression of influenza virus replication by Ko-Ken Tang through inhibition of the phosphatidylinositol 3-kinase/ Akt signaling pathway and viral RNP nuclear export. J Ethnopharmacol. 2011; 134:614-623.

24. Cheng HM, Li CC, Chen CY, Lo HY, Cheng WY, Lee $\mathrm{CH}$, Yang SZ, Wu SL, Hsiang CY, Ho TY. Application of bioactivity database of Chinese herbal medicine on the therapeutic prediction, drug development, and safety evaluation. J Ethnopharmacol. 2010; 132:429-437.

25. Zhang H, Chen Q, Zhou W, Gao S, Lin H, Ye S, Xu Y, Cai J. Chinese medicine injection shuanghuanglian for treatment of acute upper respiratory tract infection: A systematic review of randomized controlled trials. Evid Based Complement Alternat Med. 2013; 2013:987-326.

26. Shen SY, Liu JH, Tian YR, Guo J, Feng JZ, Liu SD, Zeng XJ, Dong XH, Long M. Antiviral activity of shuanghuanglian tablet against influenza A1 virus FM1 and adenovirus ADV3 in mice. China Practical Medicine. 2008; 3:50-52.
27. Wu JR, Zhang D, Zhang XM, Zhang B. Systematic review of Shuanghuanglian injection in the treatment of acute upper respiratory infection. Chinese Journal of Pharmacoepidemiology. 2016; 25:269-274. (in Chinese)

28. Ma Q, Liang D, Song S, Yu Q, Shi C, Xing X, Luo JB. Comparative study on the antivirus activity of ShuangHuang-Lian injectable powder and its bioactive compound mixture against human adenovirus III in vitro. Viruses. 2017; 9:79-91.

29. Fang L, Gao Y, Liu F, Hou R, Cai RL, Qi Y. Shuanghuang-lian attenuates lipopolysaccharide-induced acute lung injury in mice involving anti-inflammatory and antioxidative activities. Evid Based Complement Alternat Med. 2015; 2015:283939.

30. Wang J, Li FW, Zeng KZ, Li Q, Zhao XF, Zheng XH. Bioactive compounds of Shuang-Huang-Lian prescription and an insight into its binding mechanism by beta2 -adrenoceptor chromatography coupled with site-directed molecular docking. J Sep Sci. 2017; 40:4357-4365.

31. Ma CH, Ma ZQ, Fu Q, Ma SP. Ma Huang Tang ameliorates asthma though modulation of Th1/Th2 cytokines and inhibition of Th17 cells in ovalbuminsensitized mice. Chin J Nat Med. 2014; 12:361-366.

32. Wang JW, Chiang MH, Lu CM, Tsai TH. Determination the active compounds of herbal preparation by UHPLC-MS/MS and its application on the preclinical pharmacokinetics of pure ephedrine, single herbal extract of Ephedra, and a multiple herbal preparation in rats. $\mathrm{J}$ Chromatogr B Analyt Technol Biomed Life Sci. 2016; 1026:152-161

33. Wang C, Cao B, Liu QQ, et al. Oseltamivir compared with the Chinese traditional therapy maxingshigan-yinqiaosan in the treatment of H1N1 influenza: A randomized trial. Ann Intern Med. 2011; 155:217-225.

34. Hu JM, Yan JY, Cheng GZ, Wang HH, Feng CB, Zhao AX. Clinical research for Maxingshigan decoction on treating pregnancy influenza. Chinese Medicine Modern Distance Education of China. 2012; 10:80-81. (in Chinese)

35. Hsieh CF, Lo CW, Liu CH, Lin S, Yen HR, Lin TY, Horng JT. Mechanism by which ma-xing-shi-gan-tang inhibits the entry of influenza virus. J Ethnopharmacol. 2012; 143:57-67.

36. Lin $\mathrm{YC}$, Chang $\mathrm{CW}, \mathrm{Wu} \mathrm{CR}$. Antitussive, anti-pyretic and toxicological evaluation of Ma-Xing-Gan-Shi-Tang in rodents. BMC Complement Altern Med. 2016; 16:456464.

37. Ma LQ, Pan CS, Yang N, Liu YY, Yan L, Sun K, Wei XH, He K, Xiao MM, Fan JY, Han JY. Posttreatment with Ma-Xing-Shi-Gan-Tang, a Chinese medicine formula, ameliorates lipopolysaccharide-induced lung microvessel hyperpermeability and inflammatory reaction in rat. Microcirculation. 2014; 21:649-663.

38. Tao Z, Meng X, Han YQ, Xue MM, Wu S, Wu P, Yuan Y, Zhu Q, Zhang TJ, Wong CCL. Therapeutic mechanistic studies of ShuFengJieDu capsules in an acute lung injury animal model using quantitative proteomics technology. J Proteome Res. 2017; 16:4009-4019.

39. Xu YL, Zhang HH, Xue YL, YANG JS, Zhou XM, Mao B, Fan FY, Jin WL, Liu FY, Tian ZJ, Xing ZH. Phase IV clinical trial of Shufeng Jiedu Capsules in the treatment of 2031 cases of acute upper respiratory infection of windheat syndrome. China Journal of Traditional Chinese Medicine and Pharmacy. 2016; 32:353-355. (in Chinese)

40. Yang X, Yang XW. Observation on efficacy of Shufengjiedu capsules in treatment of acute suppurative 
tonsillitis. Evaluation and Analysis of Dug-Use in Hospitals of China. 2017; 17:57-59. (in Chinese)

41. Liu Y, Shi H, Jin YH, Gao YJ, Shi YJ, Liu FZ, Guo SS, Cui XL. Experimental pharmacodynamic research in vivo of Shufengjiedu capsule on treatment and prevention of influenza. World Journal of Integrated Traditional and Western Medicine. 2010; 5:107-110. (in Chinese)

42. Lv WW, Zhu TN, Qiu H, Hu T, Huang SH. Pharmacodynamic study on antiviral and antibacterial effects of Shufeng Jiedu capsules in vitro. Traditional Chinese Drug Research \& Clinical Pharmacology. 2013; 24:234-238. (in Chinese)

43. Tao Z, Gao J, Zhang G, Xue M, Yang W, Tong C, Yuan Y. Shufeng Jiedu Capsule protect against acute lung injury by suppressing the MAPK/NF-kappaB pathway. Biosci Trends. 2014; 8:45-51.

44. Ding Y, Zeng L, Li R, Chen Q, Zhou B, Chen Q, Cheng PL, Yutao W, Zheng J, Yang Z, Zhang F. The Chinese prescription lianhuaqingwen capsule exerts anti-influenza activity through the inhibition of viral propagation and impacts immune function. BMC Complement Altern Med. 2017; 17:130

45. Duan ZP, Jia ZH, Zhang J, Liu S, Chen Y, Liang LC, Zhang CQ, Zhang Z, Sun Y, Zhang SQ, Wang YY, Wu YL. Natural herbal medicine Lianhuaqingwen capsule anti-influenza A (H1N1) trial: A randomized, double blind, positive controlled clinical trial. Chin Med J (Engl). 2011; 124:2925-2933.

46. Feng GZ, Zhou F, Huang M, Yao K. Inhibitory effects of Reduning injection on human rhinoviruses in vitro. Journal of China Pharmaceutical University. 2008; 39:262-266. (in Chinese)

47. Zhang X, Gu J, Cao L, Li N, Ma Y, Su Z, Ding G, Chen L, Xu X, Xiao W. Network pharmacology study on the mechanism of traditional Chinese medicine for upper respiratory tract infection. Mol Biosyst. 2014; 10:25172525.

48. Tang LP, He RR, Li YF, Li HB, Yao XS, Hiroshi K, Xiao W. Study on antipyretic effect of Reduning injection on lipopolysaccharide-induced fever rats. China Journal of Chinese Materia Medica. 2013; 38:2374-2377. (in Chinese)

49. Liu Y, Huang Y, Wei B, Liu X, Zhang Y, Huang X, Tan Y, Sun Z. Efficacy and safety of clearing heat and detoxifying injection in the treatment of influenza: a randomized, double-blinded, placebo-controlled trial. Evid Based Complement Alternat Med. 2014; 2014:1-8.

50. Li YR, Sun SG. Revaluating the rationality and safety of Reduning injections in clinical use: An integrated approach. Chinese Journal of Clinical Pharmacology. 2015; 31:569-572. (in Chinese)

51. Ko R. A U.S. perspective on the adverse reactions from traditional Chinese medicines. J Chin Med Assoc. 2004; 67:109-116.

52. Shaw D. Toxicological risks of Chinese herbs. Planta Med. 2010; 76:2012-2018.

53. Li H, Wang S, Yue Z, Ren X, Xia J. Traditional Chinese herbal injection: Current status and future perspectives. Fitoterapia. 2018; 129:249-256.

54. Qiu F, Hou XL, Takahashi K, Chen LX, Azuma J, Kang $\mathrm{N}$. Andrographolide inhibits the expression and metabolic activity of cytochrome P450 3A4 in the modified Caco-2 cells. J Ethnopharmacol. 2012; 141:709-713.

55. Gu LL, Zhang XY, Xing WM, Xu JD, Lu H. Andrographolide-induced apoptosis in human renal tubular epithelial cells: Roles of endoplasmic reticulum stress and inflammatory response. Environ Toxicol Pharmacol. 2016; 45:257-264.

56. Anderson GD. Pregnancy-induced changes in pharmacokinetics: A mechanistic-based approach. Clin Pharmacokinet. 2005; 44:989-1008.

57. Pavek P, Smutny T. Nuclear receptors in regulation of biotransformation enzymes and drug transporters in the placental barrier. Drug Metab Rev. 2014; 46:19-32.

58. Myllynen P, Vahakangas K. Placental transfer and metabolism: An overview of the experimental models utilizing human placental tissue. Toxicol In Vitro. 2013; 27:507-512.

59. Wrobel MH, Mlynarczuk J. Chlorinated insecticides (toxaphene and endrin) affect oxytocin, testosterone, oestradiol and prostaglandin secretion from ovarian and uterine cells as well as myometrial contractions in cow in vitro. Chemosphere. 2018; 198:432-441.

60. Cao L, Pu J, Cao QR, Chen BW, Lee BJ, Cui JH. Pharmacokinetics of puerarin in pregnant rats at different stages of gestation after oral administration. Fitoterapia. 2013; 86:202-207.

61. Tracy TS, Venkataramanan R, Glover DD, Caritis SN, National Institute for Child H, Human Development Network of Maternal-Fetal-Medicine U. Temporal changes in drug metabolism (CYP1A2, CYP2D6 and CYP3A Activity) during pregnancy. Am J Obstet Gynecol. 2005; 192:633-639.

62. Zhao J, Wen LZ, Chen SH, Ling XZ. Effects of Chinese medicine Jinyebaidu Granules on immunity and genitality of pregnant mice. Chinese Traditional Patent Medicine. 2003; 25:130-133. (in Chinese)

63. Zhu J, Qiu J, Zhu Y, MA XL, Wan X, Zhang T. Teratogenicity of forsythoside gelsiccation powder in SD rats. Chinese Journal of New Drugs. 2008; 17:570-573. (in Chinese)

64. Wang L, Cheng L, Yuan Q, Cui X, Shang H, Zhang B, Li Y. Adverse drug reactions of Shuanghuanglian injection: A systematic review of public literatures. J Evid Based Med. 2010; 3:18-26.

65. Wang HS, Cheng F, Shi YQ, Li ZG, Qin HD, Liu ZP. Hypotensive response in rats and toxicological mechanisms induced by shuanghuanglian, an herbal extract mixture. Drug Discov Ther. 2008; 6:339-343.

66. Gao Y, Hou R, Han Y, Fei Q, Cai R, Qi Y. ShuangHuang-Lian injection induces an immediate hypersensitivity reaction via C5a but not IgE. Sci Rep. 2018; 8:3572-3582.

67. Ma AT, Zhong XH, Liu ZM, Shi WY, Du J, Zhai XH, Zhang T, Meng LG. Protective effects of baicalin against bromocriptine induced abortion in mice. Am J Chin Med. 2009; 37:85-95.

68. Wang YH, Song J, Dong JP, Yang TT, Hao M. Effects of baicalin on mitochondria apoptotic pathway of trophoblast cells in a preeclampsia rat model. Chinese Journal of Perinatal Medicine. 2016; 12:933-939. (in Chinese)

69. Teschke R, Wolff A, Frenzel C, Schulze J. Review article: Herbal hepatotoxicity--An update on traditional Chinese medicine preparations. Aliment Pharmacol Ther. 2014; 40:32-50.

70. Li HJ, Song DR. Effect of shuanghuanglian freeze-dried powder on the contraction of isolated human uterine smooth muscle in pregnancy. Lishizhen Medicine and Materia Medica Research. 2017; 28:772-773. (in Chinese)

71. Song DR, Zhang W, Zhao LY, Guo J, Li HJ, Du WX. 
Evaluation of embryo toxicity of Shuanghuanglian based on human placental barrier model. Chinese Journal of Pharmacology and Toxicology. 2017; 31:649-654. (in Chinese)

72. Chen JX, Xue HJ, Ye WC, Fang BH, Liu YH, Yuan SH, Yu P, Wang YQ. Activity of andrographolide and its derivatives against influenza virus in vivo and in vitro. Biol Pharm Bull. 2009; 32:1385-1391.

73. Mantani N, Andoh T, Kawamata H, Terasawa K, Ochiai H. Inhibitory effect of Ephedrae herba, an oriental traditional medicine, on the growth of influenza $\mathrm{A} / \mathrm{PR} / 8$ virus in MDCK cells. Antiviral Res. 1999; 44:193-200.

74. Hayashi K, Imanishi N, Kashiwayama Y, Kawano A, Terasawa K, Shimada Y, Ochiai H. Inhibitory effect of cinnamaldehyde, derived from Cinnamomi cortex, on the growth of influenza A/PR/8 virus in vitro and in vivo. Antiviral Res. 2007; 74:1-8.

75. Palamara AT, Nencioni L, Aquilano K, De Chiara G, Hernandez L, Cozzolino F, Ciriolo MR, Garaci E. Inhibition of influenza A virus replication by resveratrol. J Infect Dis. 2005; 191:1719-1729.

76. Law AH, Yang CL, Lau AS, Chan GC. Antiviral effect of forsythoside A from Forsythia suspensa (Thunb.) Vahl fruit against influenza A virus through reduction of viral M1 protein. J Ethnopharmacol. 2017; 209:236-247.

77. Kong WJ, Zhao YL, Shan LM, Xiao XH, Guo WY. Thermochemical studies on the quantity-antibacterial effect relationship of four organic acids from Radix Isatidis on Escherichia coli growth. Biol Pharm Bull. 2008; 31:1301-1305.

78. Pan XL, Cao X, Li N, Xu YM, Wu QY, Bai J, Yin ZM, Luo L, Lan L. Forsythin inhibits lipopolysaccharideinduced inflammation by suppressing JAK-STAT and p38 MAPK signalings and ROS production. Inflamm Res. 2014; 63:597-608.

79. Lu CN, Yuan ZG, Zhang XL, Yan R, Zhao YQ, Liao M, Chen JX. Saikosaponin a and its epimer saikosaponin d exhibit anti-inflammatory activity by suppressing activation of NF-kappaB signaling pathway. Int Immunopharmacol. 2012; 14:121-126.

80. Wang W, Wang J, Dong SF, Liu CH, Italiani P, Sun SH, $\mathrm{Xu}$ J, Boraschi D, Ma SP, Qu D. Immunomodulatory activity of andrographolide on macrophage activation and specific antibody response. Acta Pharmacol Sin. 2010; 31:191-201.

81. Cheng BH, Chan JY, Chan BC, Lin HQ, Han XQ, Zhou
X, Wan DC, Wang YF, Leung PC, Fung KP, Lau CB. Structural characterization and immunomodulatory effect of a polysaccharide HCP-2 from Houttuynia cordata. Carbohydr Polym. 2014; 103:244-249.

82. Shang XF, Pan H, Li MX, Miao XL, Ding H. Lonicera japonica Thunb.: Ethnopharmacology, phytochemistry and pharmacology of an important traditional Chinese medicine. J Ethnopharmacol. 2011; 138:1-21.

83. Hossain MS, Urbi Z, Sule A, Hafizur Rahman KM. Andrographis paniculata (Burm. f.) Wall. ex Nees: A review of ethnobotany, phytochemistry, and pharmacology. Scientific World Journal. 2014; 2014:128.

84. Li-Weber M. New therapeutic aspects of flavones: The anticancer properties of Scutellaria and its main active constituents Wogonin, Baicalein and Baicalin. Cancer Treat Rev. 2009; 35:57-68.

85. Shingnaisui K, Dey T, Manna P, Kalita J. Therapeutic potentials of Houttuynia cordata Thunb. against inflammation and oxidative stress: A review. J Ethnopharmacol. 2018; 220:35-43.

86. Al-Salihi, Bahia. Ma Huang (Ephedrae Herba): Setting the record straight. Journal of Chinese Medicine 2016;18-30.

87. Peng W, Qin RX, Li XL, Zhou H. Botany, phytochemistry, pharmacology, and potential application of Polygonum cuspidatum Sieb.et Zucc.: A review. J Ethnopharmacol. 2013; 148:729-745.

88. Xu YL, Xue YL, Zhang HH, Lv FY, Tian ZJ, Xing ZH, LiangJin W, Li XY. Clinical observation on treatment of acute upper respiratory infection of Wind-heat Syndrome with Shufeng Jiedu capsules: A randomize-controlled double-blind test. Journal of Traditional Chinese Medicine. 2015; 56:676-679. (in Chinese)

89. Saxena RC, Singh R, Kumar P, Yadav SC, Negi MP, Saxena VS, Joshua AJ, Vijayabalaji V, Goudar KS, Venkateshwarlu K, Amit A. A randomized double blind placebo controlled clinical evaluation of extract of Andrographis paniculata (KalmCold) in patients with uncomplicated upper respiratory tract infection. Phytomedicine. 2010; 17:178-185.

90. Yu DC, Chen B. Clinical observation of exogenous fever cured by Zheng Chai Hu instant granules. Guide of China Medicine. 2008; 6:124-125. (in Chinese)

(Received December 17, 2018; Revised March 11, 2019; Accepted March 12, 2019) 